\title{
Ectopic Pregnancy and Tubal Abortion
}

\author{
Madu AE, Guirguis M \\ Department of Obstetrics and Gynaecology, North Tyneside General, Hospital, Northshields, United Kingdom
}

Received: March 5, 2014; Accepted: September 12, 2014

Dear Editor,

Ectopic pregnancy (EP) is implantation of the fertilised egg occurring outside the uterine cavity accounting for $11.5 / 1000$ pregnancies in the UK. It appears to have doubled in the last decade due in part to improved diagnosis. In communities where tubal disease, salpingitis and tubal sterilization are more prevalent, the incidence of EP is higher. EP still accounts for $4 \%$ of maternal deaths; 4 deaths $/ 10000$ EPs. ${ }^{1}$ Tubal EP accounts for $95-97 \%$ of all Eps, ${ }^{1,2,4,6}$ with abdominal, ovarian and cervical accounting for $1.4 \%, 0.2 \%$ and $0.2 \%$ respectively. ${ }^{3}$ About $55 \%$ of tubal EPs occur in the ampulla region of the fallopian tube, $25 \%$ at the isthmus part, $17 \%$ at the fimbrial part and $2 \%$ at the interstitial part. ${ }^{2}$ Tubal abortion is a type of ectopic pregnancy in which an embryo, ectopically implanted in the fallopian is expelled from the uterine tube into the peritoneal cavity. It is often accompanied by significant internal bleeding, causing acute abdominal and pelvic pain. Also it may be asymptomatic, the products of conception being resorbed. Rarely the conceptus re-implants on the peritoneum and continues growing to become an abdominal pregnancy.

A 36 years nullipara presented with constant lower abdominal pain. The pain was not getting worse and there were no other constitutional symptoms described. Her last menstrual period was 6 week prior to her presentation and she had positive pregnancy test. Her past history included a surgical termination of pregnancy at 6 weeks gestation and laparoscopic cholecystectomy 2 years prior. She was known to be allergic to penicillin, erythromycin, nitrofurantion and cotrimoxazole. On examination, she was alert and not in distress. Abdominal examination was unremarkable. There were no adnexal tenderness or

\section{CORRESPONDENCE}

Dr Anthony Emeka Madu

Department of Obstetrics and Gynaecology,

North Tyneside General Hospital, Northshields, United Kingdom.

Email: emymadu@yahoo.co.uk

Phone: +447931626315 masses and no cervical excitation tenderness. Triple vaginal swabs were performed. She was admitted for observation pending results of urgent ultrasound scans, quantitative serum Bhcg estimation, full blood count, C-reactive protein (CRP), urea and electrolyte. Urgent scan results showed endometrial thickness of $15.1 \mathrm{~mm}$, bulky uterus, right ovary was not positively identified, there was an echogenic shadow posterior to the uterus, measuring $37.8 \mathrm{~mm} \mathrm{x} 48 \mathrm{~mm}$. While sitting down waiting for a clinical review after the scan, she complained of sudden onset shoulder tip pain, then collapsed and became haemodynamically unstable. Her haemoglobin $(\mathrm{Hb})$ dropped from 12.7 $\mathrm{g} / \mathrm{dl}$ at presentation to $8.2 \mathrm{~g} / \mathrm{dl}$ after her sudden collapse. She had emergency resuscitation. We attempted diagnostic laparoscopy, which was unsuccessful due to unsuccessful pneumoperitoneum. We then performed laparotomy. Operative findings at laparotomy were; about $250 \mathrm{mls}$ of fresh blood and about $800 \mathrm{mls}$ of clots in the pelvic cavity and Pouch of Douglas, normal looking tubes and ovaries. No bleeding points were noted on the fallopian tubes. She had 2 units of packed cells peri-operatively and her $\mathrm{Hb}$ increased from $10.9 \mathrm{~g} / \mathrm{dl}$ to $11.5 \mathrm{~g} / \mathrm{dl}$ prior to discharge. Her B-hcg fell dramatically from $5225 \mathrm{iu} / \mathrm{L}$ pre-operatively to $449 \mathrm{iu} / \mathrm{L} 48$ hours after surgery. B-hcg was then less than $100 \mathrm{iu} / \mathrm{L}$ one week after surgery. CRP and vaginal swab results were unremarkable.

The natural progressions of tubal EP are as follows; spontaneous involution (absorption, tubal blood mole or carneous mole) in which the conceptus dies without any symptoms, with a small bleed around it simulating caneous mole in the uterus; unruptured tubal EP; ruptured EP with dramatic signs and symptoms; tubal abortion which can be complete or incomplete and occurs when whole or part of the conceptus is expelled in to the peritoneal cavity; and abdominal pregnancy $(2-20 \%)$ which is rare, may 
die and mummifies forming lithopaedion, or may continue to grow with a high mortality and morbidity rates. Tubal abortion is almost always secondary to primary tubal EP. ${ }^{3,4,6}$ Complete tubal abortion creates a diagnostic anxiety as the tubes could appear normal as in this case without any evidence of bleeding from the tubal ostium or pathological anatomical changes around the tubal ostium. Severe intraperitoneal haemorrhage in the absence of tubal abnormality had resulted in seeking a surgical opinion to reduce the chance of missing any other incidental, unexpected and potentially surgical cause of haemoperitoneum and sudden collapse.

Tubal abortion occurs when an ampullary tubal EP separates and is expelled through the tubal ostium into the peritoneal cavity. Blood escapes from the tube and collects at in the recto-vaginal pouch of Douglas forming a pelvic haematocoele. The latter may be walled off by adhesion forming a cystic swelling behind the uterus as in this case. This was seen in the index patient as an echogenic shadow of $37.8 \mathrm{~mm} \mathrm{x}$ $48 \mathrm{~mm}$ on pelvic USS. With larger haematocoele as in the index patient, the cervix may be pushed upwards and forward as was noted in this patient simulating a retroverted uterus. Occasionally urine retention may occur. The haemorrhage noted in this patient was more active and severe, and clinical picture dramatic and startling making this case different. Her recovery was also dramatic. Thus tubal abortion can present with potentially lethal haemorrhage as in our patient.

\section{DISCLOSURE}

The authors report no conflicts of interest in this work. No violation of human rights and safety.

Funding: Nil

\section{REFERENCES}

1. Arulkuramaran S, Symonds IM, Fowlie A. Oxford Handbook of Obstetrics and Gynaecology. 1st ed. Oxford: Oxford University Press; 2004. p. 514-7.

2. Decherney AH, Nathan L. Current Obstetrics and Gynaecologic Diagnosis and Treatment. 9th ed. New York: Appleton and Lange; 1991. p. 278.

3. Shaw RW, Soutter WP, Stanton SL. Gynaecology. 3rd ed London UK: Churchill Livingstone; 2003. p. 374-5.
4. Keith-Edwards D. Dewhurst's Textbook of Obstetrics and Gynaecology for Postgraduates. 6th ed. London: Blackwell Science; 1999. p.66-70

5. Stirrat GM, Mills MS. Notes on Obstetrics and Gynaecology. 1st ed. Edinburgh: Churchill Livingstone; 2003. p. 21.

6. Hamilton-Fairley D. Lecture notes Obstetrics and Gynaecology. 1st ed. Oxford: Blackwell Science; 2004. p. $100-2$. 